3. Landau DA, Tausch E, Taylor-Weiner AN, et al. Mutations driving CLL and their evolution in progression and relapse. Nature. 2015;526(7574):525-530

4. Mansouri L, Sutton LA, Ljungstrom V, et al. Functional loss of IkappaBepsilon leads to NF-kappaB deregulation in aggressive chronic lymphocytic leukemia. J Exp Med. 2015;212(6):833-843.

5. Puente XS, Bea S, Valdes-Mas R, et al. Non-coding recurrent mutations in chronic lymphocytic leukaemia. Nature. 2015;526(7574):519-524

6. Rosenquist R, Ghia P, Hadzidimitriou A, et al. Immunoglobulin gene sequence analysis in chronic lymphocytic leukemia: updated ERIC recommendations. Leukemia. 2017;31(7):1477-1481.

7. Sutton LA, Hadzidimitriou A, Baliakas $\mathrm{P}$, et al. Immunoglobulin genes in chronic lymphocytic leukemia: key to understanding the disease and improving risk stratification. Haematologica. 2017;102(6):968-971

8. Byrd JC, O'Brien S, James DF. Ibrutinib in relapsed chronic lymphocytic leukemia. N Engl J Med. 2013;369(13):1278-1279.

9. Caligaris-Cappio F, Bertilaccio MT, Scielzo C. How the microenvironment wires the natural history of chronic lymphocytic leukemia.
Semin Cancer Biol. 2014;24:43-48.

10. Johnson AJ, Lucas DM, Muthusamy N, et al. Characterization of the TCL-1 transgenic mouse as a preclinical drug development tool for human chronic lymphocytic leukemia. Blood. 2006;108(4):13341338

11. Patrussi L, Capitani N, Ulivieri C, et al. p66Shc deficiency in the Emu-TCL1 mouse model of chronic lymphocytic leukemia enhances leukemogenesis by altering the chemokine receptor landscape. Haematologica. 2019;104(10):2040-2052.

12. Capitani N, Lucherini OM, Sozzi E, et al. Impaired expression of p66Shc, a novel regulator of B-cell survival, in chronic lymphocytic leukemia. Blood. 2010;115(18):3726-3736.

13. Patrussi L, Capitani N, Cattaneo F, et al. p66Shc deficiency enhance CXCR4 and CCR7 recycling in CLL B cells by facilitating their dephosphorylation-dependent release from beta-arrestin at early endosomes. Oncogene. 2018;37(11):1534-1550.

14. Cattaneo F, Patrussi L, Capitani N, et al. Expression of the p66Shc protein adaptor is regulated by the activator of transcription STAT4 in normal and chronic lymphocytic leukemia B cells. Oncotarget. 2016;7(35):57086-57098.

\title{
Hereditary thrombotic thrombocytopenic purpura
}

\author{
Marie Scully \\ Department of Haematology, UCLH and Cardiometabolic Programme-NIHR UCLH/UC BRC London, UK \\ E-mail: MARIE SCULLY - m.scully@nhs.net
}

doi:10.3324/haematol.2019.225896

T he first description of thrombotic thrombocytopenic purpura (TTP) by Moschowitz was published nearly 100 years ago. ${ }^{1}$ This was likely to have been an immune-mediated TTP episode and the author described multi organs affected with worsening, untreated disease. Accounts of hereditary TTP were otherwise acknowledged to be Upshaw Shulman syndrome. In 1960, Schulman reported an 8-year old girl who had repeated episodes of thrombocytopenia and hemolytic anemia from infancy. Treatment with plasma was associated with normalization of the platelet count and resolution of hemolysis, and remission was maintained with prophylactic plasma every 1-2 weeks. ${ }^{2}$ Upshaw presented a 16-year old girl with relapsing hemolytic anemia and thrombocytopenia since infancy. The patient responded to blood transfusions. During the next 11 years, Upshaw treated 32 episodes of thrombocytopenia and microangiopathic hemolysis with plasma infusions. The acute episodes invariably had a trigger, such as a minor infection, surgical procedure, pregnancy, or pancreatitis. Acute intervals lasted from three weeks to 20 months, at which time the platelet count was normal and there was a compensated hemolysis. Between these acute episodes, it was observed that intravascular platelet and red cell survival was shortened; these abnormalities normalized after the infusion of two units of plasma. ${ }^{3}$

In this edition of Haematologica, Van Dorland et al. ${ }^{4}$ present an international collaborative study on hereditary TTP. As an ultra-rare disorder, collection of meaningful data is critical to understand the clinical features of this condition, the therapy, and the long-term impact. The international registry presents data from over an 11-year period, incorporating 123 patients from four continents who presented the disease from the neonatal period, up to the seventh decade of life. We know, from numerous publications relating to the mutations identified in hereditary TTP, that there is a heterogenous distribution throughout the ADAMTS 13 gene. ${ }^{5-8}$ There are, however, two specific variants that have been identified at increased frequency in hereditary TTP.
R1060W, exon 24, is prominent in Caucasians presenting with late onset congenital TTP specifically associated with pregnancy. ${ }^{9,10}$ Within the international hereditary TTP registry, c.4143_4144dupA (exon 29; p.Glu1382Argfs*6) was prevalent, specifically within northern Europe, and was initially described at increased prevalence from central Norway. ${ }^{11}$ Further cases were documented in the international registry, confirming that those patients with compound heterozygous mutations were more likely to have an earlier presentation, specifically in the neonatal period. There were other mutations which have been identified in more than one individual in the international registry. This allows us to predict the impact of such variations within the ADAMTS 13 gene with respect to clinical features. Such observations are only possible within the breadth of a registry. ${ }^{4}$

The international registry has identified the significant delay in diagnosis of hereditary TTP; overall median age of overt presentation was 4.5 years but the clinical diagnosis was not made until a median of 16.7 years. Acute TTP episodes occurred at a median rate of 0.1 per year, ranging up to nine per year, and triggers included infections, childbirth, trauma, and in males, excess alcohol intake. The median time to resolution following an acute presentation was seven days.

There was an equal male to female ratio; this is in contrast to immune-mediated disease, which has a female preponderance. Residual ADAMTS 13 activity could not reliably predict age of onset or, indeed, disease severity, despite a cut-off of $1 \%$. This may also help to explain why patients with identical genetic variants have significant differences in the clinical phenotype. What is particularly striking and important is the clinical impact of hereditary TTP: 1) the degree of end-organ damage symptoms, either at presentation or as complications of the disease; and 2) the proportion of patients with arterial thromboembolic events, present in 28\% and covering all age groups. This was especially striking in $40-50$ years old; $>50 \%$ of these patients had at least one arterial thromboembolic event. 


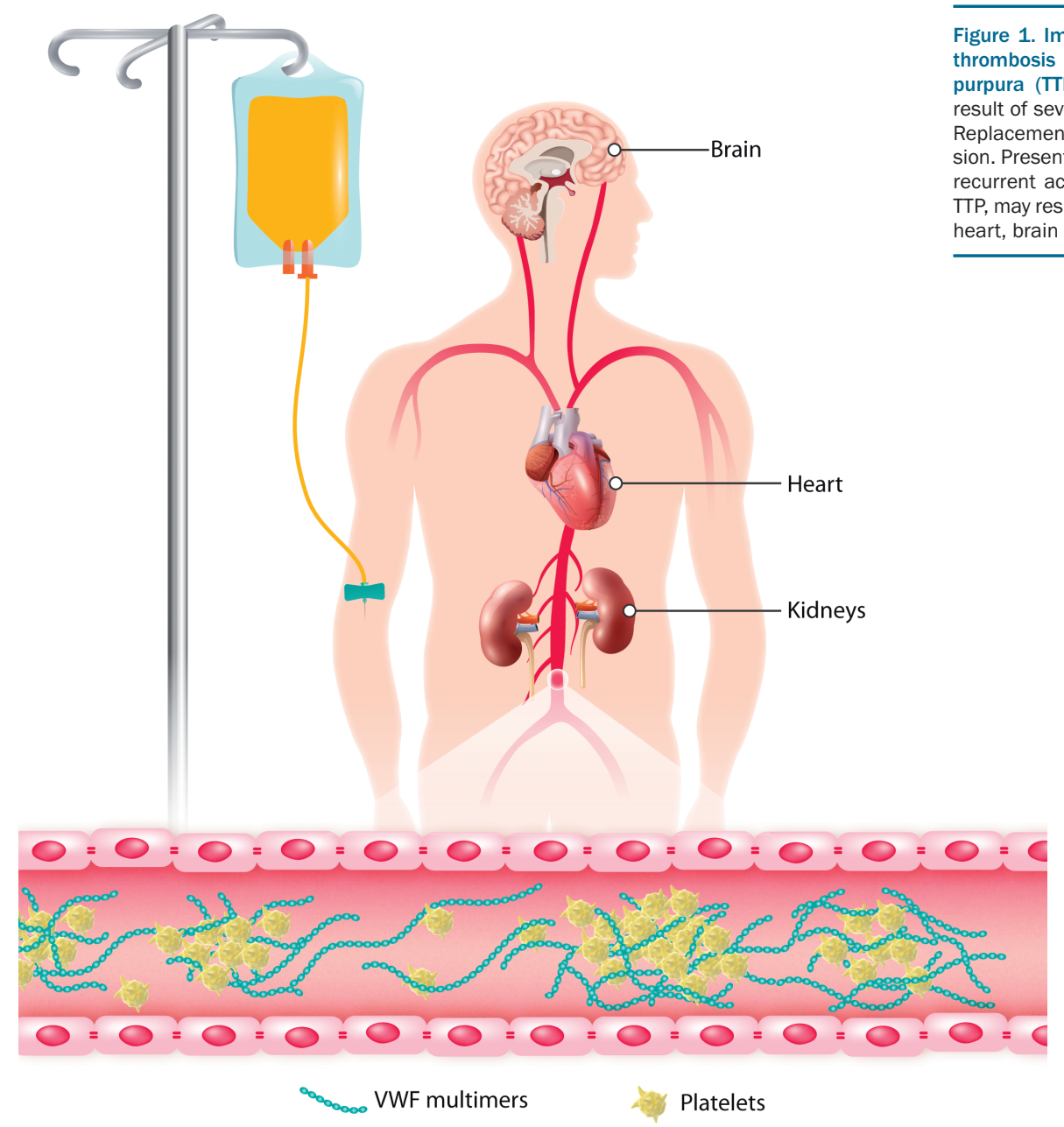

Other neurological complications include epilepsy, headaches, and a significant history of psychiatric symptoms. Renal impairment occurred in $26 \%$, some patients requiring renal replacement therapy or renal transplant and nearly $50 \%$ experiencing jaundice/liver disease.

Complimentary findings relating to the longer-term impact of hereditary TTP on end-organ damage were captured within the UK TTP registry. Furthermore, the latter cohort identified non-overt symptoms, including headaches, lethargy, and abdominal pain that, despite a normal platelet count, responded to regular plasma infusion. Indeed, ADAMTS 13 replacement therapy, primarily using plasma infusion, was associated with resolution of proteinuria and a significant reduction in stroke in those receiving treatment compared to patients not on a regular regime..$^{12}$ The proportion of patients on replacement therapy within the international registry was surprising. However, nearly one-third of the cohort only received treatment on demand. ${ }^{4}$ Both recent papers, therefore, raise the question of treatment in hereditary TTP. Firstly, the frequency of therapy. Despite a half-life of ADAMTS 13 of $2-4$ days,$^{13}$ in the international registry, trough levels were reached within 7-10 days. This has also been shown in a recent pharmacokinetic study in hereditary TTP. ${ }^{14}$ We need to reconsider how we treat patients, with particular attention to the dose of plasma and the frequency. The completion of the phase

I trial of recombinant ADAMTS 13, which was the first-inhuman trial, using increasing doses, offers a significant advancement in the future treatment of patients with hereditary TTP, delivering a pure form of the deficient enzyme, ADAMTS $13 .{ }^{15}$

In summary, the international registry presents the impact of hereditary TTP on end-organ damage, which is evident much earlier than expected within the general population; the most prevalent is arterial disease affecting the brain, heart and kidneys. Based on this important international collaborative study, in conjunction with other large cohorts, we must question how we should treat patients in the form of prophylactic ADAMTS 13 replacement (Figure 1). Should all patients with hereditary TTP be on prophylaxis? Do we wait for the patient to experience frequent acute episodes before initiating therapy? Given the increased risk, particularly of arterial events after the age of 40 , at what age should prophylaxis be initiated? What is the correct frequency of prophylaxis and what ADAMTS 13 activity level should we be aiming to achieve? Specific genetic variants may be the catalyst to personalized ADAMTS 13 replacement protocols. While there are many questions to be answered, without the information from important registries incorporating larger patient numbers the clinical impact of this ultra-rare disease cannot be addressed. 
Editorials

References

1. Moschowitz E. An acute febrile pleiochromic anemia with hyaline thrombosis of terminal arterioles and capillaries: An undescribed disease. Arch Intern Med.1925;36:89.

2. Schulman I, Pierce M, Lukens A, Currimbhoy Z. Studies on throwbopoiesis. I. A factor in normal human plasma required for platelet prodiction; chronic thrombocytopenia due to its deficiency. Blood. 1960;16:943-957.

3. Upshaw JD Jr. Congenital deficiency of a factor in normal plasma that reverses microangiopathic hemolysis and thrombocytopenia. N Angl J Med. 1978;298(24):1350-1352.

4. van Dorland HA, Mansouri Taleghani M, et al. The International Hereditary Thrombotic Thrombocytopenic Purpura Registry: Key findings at enrolment until 2017. Haematological. 2019;104(10):21072116.

5. Kinoshita S, Yoshioka A, Park YD, et al. Upshaw-Schulman syndrome revisited: a concept of congenital thrombotic thrombocytopenic purpura. Int J Hematol. 2001;74(1):101-108.

6. Kokame K, Matsumoto M, Soejima K, et al. Mutations and common polymorphisms in ADAMTS13 gene responsible for van Willebrand factor-cleaving protease activity. Proc Natl Acad Sci U S A. 2002;99(18):11902-11907.

7. Lotta LA, Garagiola I, Pall R, Cairo A, Peyvandi F. ADAMTS13 mutatons and polymorphisms in congenital thrombotic thrombocytopenic purpura. Hum Mutate. 2010;31(1):11-19.
8. Levy GG, Nichols WC, Liam EC, et al. Mutations in a member of the ADAMTS gene family cause thrombotic thrombocytopenic purpura. Nature. 2001;413(6855):488-494.

9. Sully M, Thomas M, Underwood M, et al. Thrombotic thrombocytopenic purpura and pregnancy: presentation, management, and subsequint pregnancy outcomes. Blood. 2014;124(2):211-219.

10. Camilleri RS, Cohen H, Mackie IJ, et al. Prevalence of the ADAMTS13 missense mutation R1060W in late onset adult thrombotic throwbocytopenic purpura. J Thromb Haemost. 2008;6(2):331-338.

11. van Krogh AS, Quist-Paulsen P, Wage A, et al. High prevalence of hereditary thrombotic thrombocytopenic purpura in central Norway: from clinical observation to evidence. J Thromb Haemost. 2016;14(1):73-82.

12. Alwan F, Vendramin C, Liesner R, et al. Characterization and treatment of congenital thrombotic thrombocytopenic purpura. Blood. 2019;133(15):1644-1651.

13. Furlan M, Robles R, Morselli B, Sandoz P, Lammle B. Recovery and half-life of won Willebrand factor-cleaving protease after plasma thereby in patients with thrombotic thrombocytopenic purpura. Thromb Haemost. 1999;81(1):8-13.

14. Taylor A, Vendramin C, Oosterholt S, Della Pasqua O, Scully M. Pharmacokinetics of plasma infusion in congenital thrombotic throwbocytopenic purpura. J Thromb Haemost. 2019;17(1):88-98.

15. Scilly M, Hubbard C, Ewenstein B. Recombinant ADAMTS 13 in thrombotic thrombocytopenic purpura. Oncoscience. 2017;4(1112):160-161.

1918

haematological | 2019; 104(10) 